\title{
The progenitors of core-collapse supernovae
}

\author{
Morgan Fraser $\dagger$, \\ School of Physics, O'Brien Centre for Science North, University College Dublin, Belfield, \\ Dublin 4, Ireland \\ email: morgan.fraser@ucd.ie
}

\begin{abstract}
Linking core-collapse SNe to their stellar progenitors is a major ongoing challenge. To date, $\mathrm{H}$ rich Type IIP SNe have been shown to come from red supergiants, while there is increasing evidence that the majority of stripped envelope SNe come from binary systems. The first candidates for failed $\mathrm{SNe}$, where a massive star collapses to form a black hole without a bright optical display have been identified, while the range of outbursts and eruptions from pre-SN stars are just beginning to be revealed.
\end{abstract}

Keywords. supernovae: general, supergiants

\section{Introduction}

The tremendous diversity observed in core-collapse supernovae ( $\mathrm{SNe}$ ) is believed to arise from the wide range of possible pre-SN progenitor configurations. The chemical composition of the ejecta, bolometric luminosity, ejected ${ }^{56} \mathrm{Ni}$ mass, explosion energy and timescales, are hence expected to be a function of the progenitor mass, radius, composition and multiplicity. While it is relatively easy to measure SN properties, it is much harder to connect these to the pre-SN progenitor characteristics. In this review, I discuss the use of archival imaging, hydrodynamic and spectroscopic modeling, and mass-loss diagnostics to probe the progenitors of core-collapse SNe.

\section{Progenitor diagnostics}

Progenitor detections Since the first identification of the progenitor of SN 1987A in archival data (West et al. 1987), around fifty core-collapse SNe have either detected progenitors, or had restrictive limits placed upon them (e.g. Van Dyk et al. 2003, Smartt et al. 2009). For each of these SNe, a pre-explosion image was available with sufficient depth and spatial resolution to potentially detect an individual massive star. In most cases, these images came from the Hubble Space Telescope (HST), which has now observed over $40 \%$ of nearby, massive, low inclination, star-forming galaxies.

To identify a progenitor candidate in pre-explosion images, an image of the SN with comparable depth and resolution is required. While some SNe have been observed with HST to this end, more often 8-m class telescopes equipped with adaptive-optics (AO) are used. Ground-based AO can match the $\sim 0.1^{\prime \prime}$ resolution of HST, albeit over a smaller field of view and at near-infrared (NIR) wavelengths. Using differential astrometry between the pre- and post-explosion images, the position of the SN can typically be localised to a few tens of milliarcseconds on a pre-explosion image. Once the SN position is determined, it is simply a matter measuring the flux from an identified, co-incident progenitor candidate, or setting an upper limit to its flux if no progenitor candidate is detected.

$\dagger$ Royal Society - Science Foundation Ireland University Research Fellow 
To infer a progenitor mass from measured photometry requires the use of either empirical relations between observed colours and effective temperature, or model atmospheres and synthetic photometry (e.g. the MARCS models; Gustafsson et al. 2008). In either case, the inferred temperature allows the bolometric correction to be estimated, and a progenitor luminosity calculated. Once the luminosity and temperature of a SN progenitor are known (or at least constrained), a zero-age main sequence (ZAMS) progenitor mass can be determined through comparison to stellar evolutionary tracks.

While in principle this is a relatively straightforward process, in practice several complications arise. If a SN progenitor is only observed in a single band, then a guess must be made as to its temperature. For Type IIP SNe, which require a progenitor with an extended $\mathrm{H}$ envelope to give their characteristic plateau, the progenitor is assumed to be a red supergiant (RSG). The precise choice of temperature will also affect the calculated luminosity, for example the bolometric correction to $V$-band changes by 1 mag when going from a $3400 \mathrm{~K} \mathrm{RSG}$, to a star that is only $200 \mathrm{~K}$ hotter. In such cases, it is prudent to be conservative, and set a restrictive upper limit to the luminosity and mass based on the largest plausible bolometric correction.

A second complication comes from the (often unknown) circumstellar extinction. While in many cases, attempts have been made to estimate the extinction local to a SN from the strength of the NaD lines (e.g. Turatto, Benetti \& Cappellaro 1997), these relations have considerable scatter for individual objects (Poznanski et al. 2011). Furthermore, dust local to the progenitor can be destroyed by the shock breakout of the SN, as was seen for SN 2012aw (Fraser et al. 2012). Measured extinction towards the SN can hence only be taken as a lower limit to the extinction towards the progenitor, and the safest approach is to fit the reddening simultaneously with the progenitor temperature (if colours are known for the progenitor). It is also important to remember that circumstellar dust around a progenitor should not be treated with standard interstellar extinction laws (such as Cardelli, Clayton \& Mathis 1989), as the dust is a spherical shell rather than a foreground screen (see Kochanek, Khan \& Dai 2012 for a comprehensive discussion of this point).

Finally, when determining the ZAMS mass of the SN progenitor from its luminosity, it is essential to make a physically meaningful comparison. For a core-collapse SN to explode the progenitor must have a massive Fe core, and so it is necessary to compare the observed luminosity of the progenitor to the pre-SN luminosity of a model as close as possible to Si-burning, regardless of its effective temperature. As was seen for SN 2011dh (Maund et al. 2011, Van Dyk et al. 2011), it is possible for stars to explode at a position in the HR diagram where stellar tracks do not end, as mass loss due to rotation or binary mass transfer can easily change the effective temperature of the progenitor. However, the final luminosity of the core should still be a good indicator of the progenitor ZAMS mass. It is also possible to calculate a conservative upper limit to the progenitor ZAMS mass by comparing to the luminosity of a model at the end of core He-burning.

The ultimate test of whether a claimed SN progenitor was indeed the star that exploded, is to observe the disappearance of that star at late times, after the SN has faded. Only a handful of progenitor candidates have been thus confirmed (Maund \& Smartt 2009; Fraser 2016), and in fact, in several cases progenitor candidates have been demonstrated to have been mis-identified (Maund et al. 2015). Late time observations also offer the possibility of detecting a surviving binary companion (even in the absence of a progenitor detection; Van Dyk, de Mink \& Zapartas 2016).

Nebular spectroscopy Once the ejecta of a SN becomes optically thin, its spectrum is dominated by a mixture of permitted and forbidden lines such as $\mathrm{Ca}, \mathrm{O}, \mathrm{Na}$ and $\mathrm{Mg}$. These lines arise from a combination of the primordial metal content of the progenitor 
envelope, the products of hydrostatic nuclear burning in the progenitor, and the products of explosive SN nucleosynthesis. It is hence possible to use line strengths during the nebular phase to estimate the SN progenitor mass (e.g. Fransson \& Chevalier 1989). In particular, the strength of the [O I] $\lambda \lambda 6300,6364$ lines depend strongly on progenitor mass.

This approach has been applied to both stripped envelope SNe (in particular for broadlined Type Ic SNe which are associated with GRBs; Mazzali et al. 2001) and for Type IIP SNe (Jerkstrand et al. 2014). Encouragingly, the results of nebular modeling have been found to be consistent with direct imaging of progenitors (Jerkstrand et al.). Similarly to direct imaging, nebular spectra for a population of Type IIP SNe again tend to favour progenitors in the mass range 10-15 $\mathrm{M}_{\odot}$ (Jerkstrand et al. 2015; Silverman et al. 2017).

Nebular spectroscopy is an attractive tool to probe core-collapse progenitors as it does not depend on pre-explosion imaging, but only requires that a SN is sufficiently bright that spectra can be taken at late times. This has proven especially useful for the class of superluminous SNe, the closest of which have been found at $\mathrm{z} \sim 0.1$ (Gal-Yam 2012). Using late time spectra, Jerkstrand, Smartt \& Heger 2016 demonstrated that the progenitors of these events are inconsistent with very massive stars exploding as pair instability supernovae (as had been proposed previously).

Lightcurve modeling Attempts to infer SN progenitor masses from SN lightcurves have met with mixed success. For $\mathrm{H}$ rich $\mathrm{SNe}$, there has been a tendency for hydrodynamic lightcurve modeling to favour higher progenitor masses, when compared to masses found from direct detections. For example, Utrobin \& Chugai (2009) found a progenitor mass of between 25 and $29 \mathrm{M}_{\odot}$ for SN 2004et from lightcurve modeling, while Crockett et al. (2011) estimated the progenitor mass to be 7 to $13 \mathrm{M}_{\odot}$ from pre-explosion imaging. Morozova et al.(2015) compared SNEC and CMFGEN models of the lightcurve resulting from the explosion of a $15 \mathrm{M}_{\odot} \mathrm{RSG}$, and find plateau durations that differ by around two weeks. The cause of such differences is not yet clear, and as noted by Dall'Ora et al. (2014) and others, further work is needed to explore the differences between various hydrodynamic codes.

Efforts to measure the ejecta mass of stripped envelope SNe have met with more success. Hydrodynamic models have been applied to Type IIb SNe such as SN 2011dh (Bersten et al. 2012), and in general these have yielded progenitor masses consistent with those found from direct imaging. Along with comprehensive hydrodynamic modeling, attempts have also been made to apply simple semi-analytic models (Arnett 1982) to large samples of stripped envelope SNe (Drout et al. 2011; Lyman et al. 2016). In general, these authors have found ejecta masses $\lesssim 2 \mathrm{M}_{\odot}$ for Ibc SNe, considerably lower than would be expected for single Wolf-Rayet stars, but consistent with the ejecta mass from stars $<15 \mathrm{M}_{\odot}$ in binaries.

Mass loss diagnostics The recent advent of wide field imaging surveys, coupled with the capability to rapidly identify and follow up transients, has opened up a new window on SNe. "Flash spectroscopy" exploits spectra taken of SNe discovered less than one day after explosion (Gal-Yam et al. 2014), when the extended progenitor wind has not yet been over-taken by the SN ejecta, and is illuminated by the shock-breakout of the SN. By modeling these spectra, which are dominated by narrow, high-ionization emission lines, it is possible to determine mass loss rates, and the abundances in the wind (Groh 2014). While this has only been done for a handful of SNe to date, this will likely increase as high cadence surveys cover progressively larger areas of the sky.

$\mathrm{X}$-rays and radio observations can also be used to constrain the pre-SN mass loss experienced by a progenitor. Dwarkadas (2014) noted that there were no x-ray bright Type IIP SNe consistent with mass loss rates above $10^{-5} \mathrm{M}_{\odot} \mathrm{yr}^{-1}$. As mass loss scales 
with luminosity, and hence with mass, they argued that this implied that most Type IIP progenitors had ZAMS masses below $19 \mathrm{M}_{\odot}$. However, as pointed out by Beasor \& Davies (2016), the significant scatter in x-ray mass loss diagnostics and in the relation between mass loss and luminosity, means that there is a large uncertainty on this limit. Similarly, radio observations can be used to probe the circumstellar environment of a SN, and hence its mass loss rate. For Type IIb SNe, Chevalier \& Soderberg (2010) suggested that radio lightcurves could be split into two classes, those coming from either extended or compact progenitors. However, in the case of SN 2011dh, the radius of the progenitor from preexplosion observations is inconsistent with that inferred from the radio (Soderberg et al. 2012).

\section{Implications for SN progenitors}

To date, the majority of detected SN progenitors have been RSGs associated with Type IIP SNe. This is unsurprising as this is the most common subtype of SNe, and RSGs are bright in the I-band filter which is frequently used for HST observations of nearby galaxies. What is more surprising is the apparent deficit of Type IIP SN progenitors above $\sim 16 \mathrm{M}_{\odot}$, given that RSGs up to about $30 \mathrm{M}_{\odot}$ are seen in the Milky Way. Unless these stars evolve back across the HR diagram before they explode (Groh et al. 2013a), then will presumably explode as Type IIP SNe. While the initial mass function favours lower mass progenitors, if we consider the 26 observed detections and upper limits to progenitors from Smartt (2015), then four of these should have been above $20 \mathrm{M}_{\odot}$. Naïvely, the most massive progenitors will also be the easiest to find, as they will be the most luminous. The absence of detected high-mass RSG progenitors has been termed the "Red Supergiant Problem" by Smartt et al. (2009).

A simple, if unexciting, explanation for the RSG problem is that the most massive progenitors are missed due to extinction. Beasor \& Davies (2016) used IR photometry of RSGs in a young cluster in the Large Magellanic Cloud to study mass-loss rates, and found that the most evolved stars in the cluster were the most reddened. From this, they suggested that reddening may explain the apparent lack of high mass SN progenitors. While this is an appealing explanation, it is not clear that it will explain all the missing RSGs. Walmswell \& Eldridge (2012) found that when they took circumstellar dust into account, the maximum mass for a Type IIP progenitor increased to only $\sim 22$ $\mathrm{M}_{\odot}$. Furthermore, even if the most massive progenitors of Type IIP SNe were dust enshrouded in pre-explosion imaging, their nebular spectra should still match a higher mass star.

The finding by Smartt et al. (2009) that there is a paucity of Type II SN progenitors with ZAMS mass $>16 \mathrm{M}_{\odot}$ raises the exciting possibility that these stars are collapsing to form black holes without a bright SN. Kochanek et al.(2008) proposed an ambitious strategy to find these failed SNe - monitor $10^{6}$ RSGs for a number of years to see if any disappear. A search of the HST archive was conducted by Reynolds, Fraser \& Gilmore (2015) for disappearing RSGs in nearby galaxies, while Gerke et al. (2015) attempted to find disappearing massive stars using regular monitoring of a sample of galaxies with 8-m telescopes. In both cases, candidates for failed SNe were found, and in one case, shown to have remained well below its original flux level some years later (Adams et al. 2015).

For stripped envelope SNe (Types IIb, Ib and Ic), it appears that binary interaction is a necessary requirement in a large fraction (or perhaps the majority) of cases. This is chiefly evidenced by the small ejecta masses seen for stripped envelope SNe (Lyman et al. 2016), along with the clear detections of IIb progenitors and their binary companions in archival imaging. The fact that only a single Ibc progenitor has been observed to date 
does not provide strong constraints on their progenitors, as most Wolf Rayet stars would be below the sensitivity of the available pre-explosion images (Eldridge et al. 2013).

While several Type IIb SNe have been linked with yellow supergiants (Maund et al. 2011, Van Dyk et al. 2011), only a single Type Ibc SN has an identified progenitor (Cao et al. 2013). The pre-explosion source found to be coincident with iPTF13bvn was originally suggested to be a Wolf-Rayet star (Groh, Georgy \& Ekström 2013), but later shown to be a more convincing match to a lower mass He star progenitor (Eldridge et al. 2015). A low mass progenitor was also favoured by hydrodynamic modeling of the SN lightcurve (Bersten et al. 2014; Fremling et al. 2014). Late time imaging reveals that the progenitor candidate identified for iPTF13bvn has faded, although whether the binary companion can now be seen remains unclear (Eldridge \& Maund 2016; Folatelli et al. 2016).

Aside from Type II and stripped envelope SNe, a handful of interacting Type IIn SNe have associated progenitor detections (e.g. Gal-Yam \& Leonard 2009). At least some of these SNe appear to come from Luminous Blue Variables (LBVs), stars which were classically not expected to explode. However, the connection between Type IIn SNe, so-called supernova impostors, and LBVs remains unclear.

\section{Future avenues for progenitor studies}

While it is tempting to expect that JWST and future $30-\mathrm{m}$ class telescopes will dramatically increase our sample of SN with progenitor detections, this will likely take some time, as new facilities must first build up an archive of deep imaging of nearby galaxies. For Type II SNe, JWST and E-ELT will allow for fainter progenitors to be detected, in principle allowing the lower mass limit for Fe-core collapse (or indeed electron capture $\mathrm{SNe}$ ) to be better constrained. Furthermore, NIR imaging (IJHK) is less affected by extinction, and can provide a sensitive test of RSG temperatures (Davies et al. 2013). While possible Wolf-Rayet progenitors of stripped envelope SNe are expected to be luminous, as they are hot they emit most of their flux in the UV (as do lower mass He-giant progenitors; Kim, Yoon \& Koo 2015). Hence JWST, or 30-m telescopes with next-generation AO working in the NIR are unlikely to lead to a progenitor detection for these classes of SNe.

While most observed SN progenitors have been identified in a single image, in a few cases we are fortunate enough to have repeated observations of the progenitor over a number of years (Fraser et al. 2014, Kochanek et al. 2017). This has enabled us to be sensitive to pre-explosion variability in some progenitors. In particular, monitoring on the timescale of years to decades is well matched to the timescales for shell C-burning and core-O burning, and can potentially probe pre-SN instabilities (Smith \& Arnett 2014). In the case of ASASSN-16fq, pre-explosion imaging limited the change in luminosity in the years prior to core-collapse to around $300 \mathrm{~L}_{\odot} \mathrm{yr}^{-1}$ or less (Kochanek et al. 2017). To date only six SNe have repeated progenitor detections, but as the number of galaxies with multiple epochs of pre-explosion imaging increases, this will improve. The Large Synoptic Survey Telescope (LSST) will play a significant role in constraining progenitor variability for host galaxies $<10 \mathrm{Mpc}$. If we consider SN 2012aw, which was at only $10 \mathrm{Mpc}$, the progenitor had an magnitude of $I=23.4$ (Fraser et al., 2012). This progenitor would have been detectable in a single epoch of LSST imaging. In combination with high spatial resolution HST or JWST imaging to disentangle the progenitor from the surrounding stellar population, LSST can in principle supply a pre-explosion lightcurve for a significant sample of nearby SNe. As an aside, it is worth noting that most SNe that are close enough to have a potential detection will likely be saturated in LSST data 
(at least at early times). Regular pre-explosion monitoring is also of interest for Type IIn SNe, which may eject material shortly before exploding.

Alongside observational effort, advances in stellar evolutionary modeling can help better understand SN progenitors. The observational evidence for binaries producing many stripped envelope SNe is consistent with the finding of Sana et al. (2012) that over 70\% of massive stars will undergo some mass transfer during their lives. If we consider that half of these stars will undergo mass loss, then we obtain a fraction of stars which is comparable to the overall relative rate of stripped envelope SNe (37\%; Smith et al. 2011). However, the implication of this is that a significant fraction of the SNe where the progenitor has retained a relatively massive $\mathrm{H}$ envelope must have been the mass-gainers in a binary system. In some cases, the progenitor may even be the product of a merger (as was proposed for SN 1987A by Podsiadlowski, Joss \& Rappaport 1990). While in most cases, the limited observational data on the progenitor does not justify the additional free parameters that a binary model would entail, as more SNe are discovered with multi-band progenitor detections, binary progenitor models will become testable.

Finally, the first detection of gravitational waves by advanced LIGO (Abbott et al. 2016) has opened up a new window on the Universe, that can potentially shed light on the mechanism of core-collapse. Unfortunately, even for an exceptionally nearby SNe such as SN 2008bk (4 Mpc), the associated gravitational wave signal is likely not to be detectable with Advanced LIGO (Ott 2009; Gossan et al. 2016). Similarly, neutrino detectors such as IceCube will be unable to detect neutrinos associated with a corecollapse at this distance (IceCube Collaboration, 2011). For now, and pending the next Galactic supernova, visible and NIR observations of nearby SNe and their host galaxies will continue to shed light on their progenitors.

\section{Acknowledgements}

I thank the organisers of IAUS 329 for hosting a stimulating and thought provoking meeting. I would also like to thank my collaborators with whom I have had the pleasure of hunting for supernova progenitors, especially Stephen Smartt, JJ Eldridge, Seppo Mattila, Nancy Elias de la Rosa, Justyn Maund, Rubina Kotak, Andrea Pastorello, Tom Reynolds, Anders Jerkstrand and Cosimo Inserra. This work has been supported by a Royal Society - Science Foundation Ireland University Research Fellowship.

\section{References}

B. P. Abbott et al. (LIGO Scientific Collaboration and Virgo Collaboration) 2016, Phys. Rev. Lett., 116, 061102

Adams, S. M., Kochanek, C. S., Gerke, J. R., Stanek, K. Z., \& Dai, X. 2016, arXiv:1609.01283

Arnett, W. D. 1982, ApJ, 253, 785

Beasor, E. R. \& Davies, B. 2016, MNRAS, 463, 1269

Bersten, M. C., Benvenuto, O. G., Nomoto, K., et al. 2012, ApJ, 757, 31

Bersten, M. C., Benvenuto, O. G., Folatelli, G., et al. 2014, AJ, 148, 68

Cao, Y., Kasliwal, M. M., Arcavi, I., et al. 2013, ApJL, 775, L7

Cardelli, J. A., Clayton, G. C., \& Mathis, J. S. 1989, ApJ, 345, 245

Chevalier, R. A. \& Soderberg, A. M. 2010, ApJL, 711, L40

Crockett, R. M., Smartt, S. J., Pastorello, A., et al. 2011, MNRAS, 410, 2767

Dall'Ora, M., Botticella, M. T., Pumo, M. L., et al. 2014, ApJ, 787, 139

Davies, B., Kudritzki, R.-P., Plez, B., et al. 2013, ApJ, 767, 3

Dwarkadas, V. V. 2014, MNRAS, 440, 1917

Eldridge, J. J., Fraser, M., Smartt, S. J., Maund, J. R., \& Crockett, R. M. 2013, MNRAS, 436, 774 
Eldridge, J. J., Fraser, M., Maund, J. R., \& Smartt, S. J. 2015, MNRAS, 446, 2689

Eldridge, J. J. \& Maund, J. R. 2016, MNRAS, 461, L117

Folatelli, G., Van Dyk, S. D., Kuncarayakti, H., et al. 2016, ApJL, 825, L22

Fransson, C. \& Chevalier, R. A. 1989, ApJ, 343, 323

Fraser, M., Maund, J. R., Smartt, S. J., et al. 2012, ApJL, 759, L13

Fraser, M., Maund, J. R., Smartt, S. J., et al. 2014, MNRAS, 439, L56

Fraser, M. 2016, MNRAS, 456, L16

Fremling, C., Sollerman, J., Taddia, F., et al. 2014, A\&SA, 565, A114

Gal-Yam, A. 2012, Science, 337, 927

Gal-Yam, A., Arcavi, I., Ofek, E. O., et al. 2014, Nature, 509, 471

Gerke, J. R., Kochanek, C. S., \& Stanek, K. Z. 2015, MNRAS, 450, 3289

Gossan, S. E., Sutton, P., Stuver, A., et al. 2016, Phys. Rev. D, 93, 042002

Groh, J. H., Meynet, G., Georgy, C., \& Ekström, S. 2013a, A $6 A$, 558, A131

Groh, J. H., Georgy, C., \& Ekström, S. 2013b, A\&A, 558, L1

Groh, J. H. 2014, A\&A, 572, L11

Gustafsson, B., Edvardsson, B., Eriksson, K., et al. 2008, A\& $A, 486,951$

IceCube Collaboration, Abbasi, R., Abdou, Y., et al. 2011, arXiv:1108.0171

Jerkstrand, A., Smartt, S. J., Fraser, M., et al. 2014, MNRAS, 439, 3694

Jerkstrand, A., Smartt, S. J., Sollerman, J., et al. 2015, MNRAS, 448, 2482

Jerkstrand, A., Smartt, S. J., \& Heger, A. 2016, MNRAS, 455, 3207

Kim, H.-J., Yoon, S.-C., \& Koo, B.-C. 2015, ApJ, 809, 131

Kochanek, C. S., Beacom, J. F., Kistler, M. D., et al. 2008, ApJ, 684, 1336-1342

Kochanek, C. S., Khan, R., \& Dai, X. 2012, ApJ, 759, 20

Kochanek, C. S., Fraser, M., Adams, S. M., et al. 2016, arXiv:1609.00022

Lyman, J. D., Bersier, D., James, P. A., et al. 2016, MNRAS, 457, 328

Maund, J. R. \& Smartt, S. J. 2009, Science, 324, 486

Maund, J. R., Fraser, M., Ergon, M., et al. 2011, ApJL, 739, L37

Maund, J. R., Fraser, M., Reilly, E., Ergon, M., \& Mattila, S. 2015, MNRAS, 447, 3207

Mazzali, P. A., Nomoto, K., Patat, F., \& Maeda, K. 2001, ApJ, 559, 1047

Morozova, V., Piro, A. L., Renzo, M., et al. 2015, ApJ, 814, 63

Ott, C. D 2009, Classical and Quantum Gravity, 26, 063001

Podsiadlowski, P., Joss, P. C., \& Rappaport, S. 1990, A\&A, 227, L9

Poznanski, D., Ganeshalingam, M., Silverman, J. M., \& Filippenko, A. V. 2011, MNRAS, 415, L81

Reynolds, T. M., Fraser, M., \& Gilmore, G. 2015, MNRAS, 453, 2885

Sana, H., de Mink, S. E., de Koter, A., et al. 2012, Science, 337, 444

Silverman, J. M., Pickett, S., Craig Wheeler, J., et al. 2017, MNRAS in press

Smartt, S., J. J., E., Crockett, R., \& Maund, J. 2009, MNRAS, 395, 1409

Smartt, S. J. 2015, PASA, 32, e016

Smith, N., Li, W., Filippenko, A. V., \& Chornock, R. 2011, MNRAS, 412, 1522

Smith, N. \& Arnett, W. D. 2014, ApJ, 785, 82

Soderberg, A. M., Margutti, R., Zauderer, B. A., et al. 2012, ApJ, 752, 78

Turatto, M., Benetti, S., \& Cappellaro, E. 2003, From Twilight to Highlight: The Physics of Supernovae, 200

Utrobin, V. P. \& Chugai, N. N. 2009, A\& $A, 506,829$

Van Dyk, S. D., Li, W., \& Filippenko, A. V. 2003, PASP, 115, 1

Van Dyk, S. D., Li, W., Cenko, S. B., et al. 2011, ApJL, 741, L28

Van Dyk, S. D., de Mink, S. E., \& Zapartas, E. 2016, ApJ, 818, 75

Walmswell, J. J. \& Eldridge, J. J. 2012, MNRAS, 419, 2054

West, R. M., Lauberts, A., Schuster, H.-E., \& Jorgensen, H. E. 1987, A\&A A, 177, L1 